\title{
VALUATION OF VARIABLE ANNUITY CONTRACTS WITH CLIQUET OPTIONS IN ASIA MARKETS
}

\author{
Ming-hua Hsieh \\ Department of Management Information Systems \\ National Chengchi University \\ NO. 64, Sec. 2, ZhiNan Rd. \\ Taipei, 11605, TAIWAN
}

\begin{abstract}
Variable annuities are very appealing to the investor. For example, in United States, sales volume on variable annuities grew to a record 184 billion in calendar year 2006. However, due to their complicated payoff structure, their valuation and risk management are challenges to the insurers. In this paper, we study a variable annuity contract with cliquet options in Asia markets. The contact has quanto feature. We propose an efficient Monte Carlo method to value the contract. Numerical examples suggest our approach is quite efficient.
\end{abstract}

\section{INTRODUCTION}

Equity-linked insurance contracts has become very popular in the market. There are two major types of equity-linked insurance contracts: variable annuity (VA) and equity-linked annuity (EIA). The markets of VA and EIA are very large. According to LIMRA International and Advantage Compendiumm, VA and EIA contracts have very high sales volume (Table 1) in United States.

Table 1: VA and EIA sales volume in billion dollars in United States, 2003-2007.

\begin{tabular}{|r|rr|}
\hline Year & VA & EIA \\
\hline 2003 & 129.4 & 14.0 \\
2004 & 132.9 & 23.4 \\
2005 & 137.6 & 27.3 \\
2006 & 184.2 & 25.3 \\
2007 & 160.4 & 25.1 \\
\hline
\end{tabular}

Compared to the traditional annuity product, VA and EIA have the advantage of offering additional return when the linked equities perform well. Although VA and EIA share this commonality, they are different in various aspects:
1. A typical (non-registered) EIA is an insurance company "general account" product. This means that EIA is treated as a liability item on insurance company's balance sheet. On the other hand, VA is a "separate account" product. Except the guarantees embedded in the contract, any gains or losses to the underlying assets in the VA separate account are reflected directly and immediately in the VA contract owners' accumulation values.

2. Owners of variable annuities are generally allowed to modify their investment allocations periodically, sometimes as often as daily, and VA contract values change according to the performance of the selected investment portfolios. Therefore, VA contracts provide their owners with considerably greater investment flexibility than do EIA contracts. The linked indices in EIA contracts are usually the well known indices such as S\&P 500. So, EIA owners basically cannot have direct influence on the values of linked indices.

3. VAs are considered to be securities and must be registered with the Securities and Exchange Commission (SEC). On contrary, EIAs are not required to register with SEC and most EIAs are not registered.

4. At maturity, the guarantees embedded in EIA contracts are usually in-the-money. On the other hand, VA's guarantees are usually out-of-the-money.

Above descriptions about VA and EIA are for US market. Asia markets for VA and EIA have a different situation. For example, equity-linked insurance contracts must be premium protected and must be "separate account" products in Taiwan. Regulator set a very high "capital requirement" standard for insurers for providing guarantees in equitylinked insurance. For this reason, insurers in Taiwan have to transfer their risks associated with these guarantees to third party. Reinsurance is one way for transferring these risks, but it is usually too expensive for insurers. A more popular 
way is to package premium-protected structure products as equity-linked insurance contracts. Such premium-protected structure products are designed and sold by investment banks. In order to be a qualified provider for such structure products, the investment bank must maintain a high credit rating. These structure products are classified as variable annuities in Taiwan, because they are separate account products. However, their financial property are more like EIA products in United States. These products have exotic payoff functions and their valuation becomes an important issue for the insurers. In this paper, we focus on one of such structure products. The variable annuity contract considered has quanto feature and embedded cliquet options. Such contracts are attractive to the investor because of their protection against downside risk and significant upside potential. We discuss the Monte Carlo valuation approach and then propose efficient algorithms. In particular, we applied variance reduction technique of control variates to improve the performance of Monte Carlo approach.

This paper is organized as follows. In Section 2, we describe the variable annuity contract under consideration. In Section 3, we first describe the stochastic models for the linked index and exchange rate. Then we present the risk neutral valuation formulas under the stochastic models. In Section 4, we devise a few control variates and test their effectiveness via numerical examples. Finally, Section 5 offers some concluding remarks.

\section{VARIABLE ANNUITY CONTRACTS WITH CLIQUET OPTIONS IN ASIA MARKETS}

The payoff of the variable annuity contracts under consideration is very similar to simple ratchet EIA contracts with maturity guarantee; see, Hardy (2003) and Hsieh and Chiu (2007) for more information. The VA contracts might also be called cliquet options with global floor (Kjaer 2006). However, there is one major difference between the VA contracts considered and simple ratchet EIA contracts: the VA contracts considered are quantos. That is, the linkedindex is dominated in a different currency. For example, the contracts pay off in Australian dollar and the linked-index is S\&P 500 which is dominated in United States dollar.

The index participation is evaluated annually. Let $T$ be the maturity of a VA contract and $S(t)$ be the linked-index at time $t \leq T$. We set

$$
R_{t}=\frac{S(t)}{S(t-1)}, \quad t=1, \ldots, T,
$$

which are the annual returns of linked-index. The effective annual returns of the VA contract are defined as

$$
\tilde{R}_{t}=1+\min \left(\max \left(\alpha\left(R_{t}-1\right), f\right), c\right)
$$

where $f$ is the annual guarantee rate, $c$ is the annual cap rate, and $\alpha$ is the participation rate in the linked-index.

Treat these effective annual returns as simple returns and sum them arithmetically, then the total return at maturity is

$$
R_{M}=1+\sum_{t=1}^{T}\left(\tilde{R}_{t}-1\right)=1-T+\sum_{t=1}^{T} \tilde{R}_{t}
$$

In addition to annual guarantee rate $f$, the VA contract also provides guarantee at maturity. This type of guarantee is sometimes called global guarantee. Let the initial investment be $P$. If the maturity guarantee promises a maturity guarantee rate $G$, then the payoff of the VA contract is

$$
P \cdot \max \left(R_{M}, G\right),
$$

\section{VALUATION FORMULAS FOR THE VA CONTRACT CONSIDERED}

Most of the previous related research in insurance field (Hardy (2004), Lee (2003), Tiong (2000), and Gerber and Shiu (2003)) adopted the Black-Scholes assumptions (Black and Scholes 1973) for the linked-index and interest rate. We follow these assumptions. But, since the VA contract is quanto, we need to add exchange rate model. More specifically, the linked-index $S(t)$ and exchange rate $C(t)$ follow geometric Brownian motions and the interest rate $r$ (local currency) and $r_{f}$ (foreign currency) are constants.

$$
\begin{aligned}
\frac{d S(t)}{S(t)} & =\mu_{S} d t+\sigma_{S} d z_{1}(t) \\
\frac{d C(t)}{C(t)} & =\mu_{C} d t+\sigma_{C}\left[\rho d z_{1}(t)+\bar{\rho} d z_{2}(t)\right] \\
\frac{d B(t)}{B(t)} & =r d t \\
\frac{d D(t)}{D(t)} & =r_{f} d t
\end{aligned}
$$

where $z_{i}(t), i=1,2$ are independent standard Brownian motions, $\sigma_{S}$ is the volatility of the linked index, $\sigma_{C}$ is the volatility of the exchange rate, $\rho$ is the correlation coefficient between $\log (S(t))$ and $\log (C(t)), \bar{\rho}=\sqrt{1-\rho^{2}}$ is the orthogonal complement of $\rho$ and $B(t)$ and $D(t)$ denote the local and foreign money market accounts, respectively. We call the models defined in (5) Black-Scholes quanto model (Baxter and Rennie 1996). To make the model more concrete, we might assume that the local current currency is Australian dollar and the foreign currency is US dollar. So, there are three tradables in Australian dollar: $B(t), C(t) D(t)$, and $C(t) S(t)$. Based on Girsanov's and martingale representation theorems (see Baxter and Rennie (1996), for example), there exists a unique measure $Q$ such that, under which, both $C(t) D(t) / B(t)$ and $C(t) S(t) / B(t)$ 
are martingales. And under measure $Q$, the processes $S(t)$ and $C(t)$ can be written as

$$
\begin{aligned}
\frac{d S(t)}{S(t)} & =\left(r_{f}-\rho \sigma_{S} \sigma_{C}\right) d t+\sigma_{S} d \tilde{z}_{1}(t) \\
\frac{d C(t)}{C(t)} & =\left(r-r_{f}\right) d t+\sigma_{C}\left[\rho d \tilde{z}_{1}(t)+\bar{\rho} d \tilde{z}_{2}(t)\right]
\end{aligned}
$$

where $\tilde{z}_{1}$ and $\tilde{z}_{2}$ are independent standard Brownian motions under measure $Q$.

If a derivative's payoff depends only on $S(t)$ and/or $C(t)$, then its fair value can be represented as expectation under measure $Q$ (Baxter and Rennie 1996). This fair value is actually equal to the initial value of a (dynamic) replicating portfolio. Such valuation approach is usually called risk neutral valuation (see, for example, Harrison and Kreps (1979) and Harrison and Pliska (1981)) and measure $Q$ is called risk neutral measure. Therefore, the fair value of the VA contract defined in Section 2 can be written as

$$
V=E_{Q}\left[e^{-r T} P \cdot \max \left(R_{M}, G\right)\right]
$$

where $E_{Q}[\cdot]$ denotes the expectation operator under measure $Q$.

\section{MONTE CARLO APPROACH}

It is well known that, under the risk neutral measure $Q$, $\log \left(R_{t}\right)$ are independent normal random variables with common mean

$$
r_{f}-\rho \sigma_{S} \sigma_{C}-\frac{\sigma_{S}^{2}}{2}
$$

and common variance $\sigma_{S}^{2}$ (Hull 2006). Since $R_{M}$ is just a function of $R_{t}, t=1, \ldots, T$. Equation (7) implies Monte Carlo approach can be used to price the contract. We shall use control variates (see, e.g., Bratley, Fox, and Schrage (1983) and Law and Kelton (2000)) to speedup the Monte Carlo method.

From Equation (7), it is easy to see that $R_{M}$ can be a good control variate. To make $R_{M}$ a valid control variate, we need to compute $E_{Q}\left[R_{M}\right]$. To this end, we follow the idea in Hsieh and Chiu (2007).

Using (2) we can get

$$
\tilde{R}_{t}=(1-\alpha)+\alpha \min \left(\max \left(f_{\alpha}, R_{t}\right), c_{\alpha}\right),
$$

where $f_{\alpha}=1+f / \alpha$ and $c_{\alpha}=1+c / \alpha$. Set

$$
X_{t}=\min \left(\max \left(f_{\alpha}, R_{t}\right), c_{\alpha}\right)
$$

Then it is easy to see that $X_{t}$ 's are independent censored lognormal random variables with censored values $f_{\alpha}$ and $c_{\alpha}$.
We use (3) to obtain

$$
\begin{aligned}
E_{Q}\left[R_{M}\right] & =E_{Q}\left[1-T+\sum_{t=1}^{T} \tilde{R}_{t}\right] \\
& =E_{Q}\left[(1-\alpha T)+\alpha T X_{1}\right] \\
& =1-\alpha T+\alpha T E_{Q}\left[X_{1}\right]
\end{aligned}
$$

Therefore, we reduce the task of computing $E_{Q}\left[R_{M}\right]$ to the task of computing $E_{Q}\left[X_{1}\right]$.

To compute $E_{Q}\left[X_{1}\right]$, we first write

$$
\begin{gathered}
E_{Q}\left[X_{1}\right]=f_{\alpha} P\left(R_{1} \leq f_{\alpha}\right)+E_{Q}\left[\left[R_{1} ; f_{\alpha} \leq R_{1} \leq c_{\alpha}\right]\right. \\
+c_{\alpha} P\left(R_{1} \geq c_{\alpha}\right) .
\end{gathered}
$$

Then, by representing $R_{1}$ as

$$
\exp \left(r_{f}-\rho \sigma_{S} \sigma_{C}-\sigma_{S}^{2} / 2+\sigma_{S} N(0,1)\right)
$$

and letting

$$
d_{1}=\frac{\log f_{\alpha}-r_{f}}{\sigma_{S}}+\frac{2 \rho \sigma_{C}+\sigma_{S}}{2},
$$

and

$$
d_{2}=\frac{\log c_{\alpha}-r_{f}}{\sigma_{S}}+\frac{2 \rho \sigma_{C}+\sigma_{S}}{2},
$$

we obtain

$$
\begin{aligned}
& P\left(R_{1} \leq f_{\alpha}\right)=P\left(N(0,1) \leq d_{1}\right)=\Phi\left(d_{1}\right) \\
& P\left(R_{1} \geq c_{\alpha}\right)=P\left(N(0,1) \geq d_{2}\right)=\Phi\left(-d_{2}\right)
\end{aligned}
$$

and

$$
\begin{aligned}
& E\left[R_{1} ; f_{\alpha} \leq R_{1} \leq c_{\alpha}\right] \\
= & \int_{d_{1}}^{d_{2}} e^{r_{f}-\rho \sigma_{S} \sigma_{C}-\sigma_{S}^{2} / 2+\sigma_{S} z} \cdot \phi(z) d z \\
= & e^{r_{f}-\rho \sigma_{S} \sigma_{C}}\left[\Phi\left(d_{2}-\sigma_{S}\right)-\Phi\left(d_{1}-\sigma_{S}\right)\right]
\end{aligned}
$$

where $\phi(\cdot)$ and $\Phi(\cdot)$ are the density function and the cumulative distribution function of standard normal random variable, respectively.

Combining these three terms, we get the explicit formula for $E_{Q}\left[X_{1}\right]$ :

$$
\begin{aligned}
E_{Q}\left[X_{1}\right]= & f_{\alpha} \Phi\left(d_{1}\right)+c_{\alpha} \Phi\left(-d_{2}\right) \\
& +e^{r_{f}-\rho \sigma_{S} \sigma_{C}}\left[\Phi\left(d_{2}-\sigma_{S}\right)-\Phi\left(d_{1}-\sigma_{S}\right)\right](
\end{aligned}
$$

With (13) and (10), the following proposition is straightforward. 
Proposition 1

$$
\begin{aligned}
& E_{Q}\left[R_{M}\right]=1-\alpha T \\
& +\alpha T\left(f_{\alpha} \Phi\left(d_{1}\right)+c_{\alpha} \Phi\left(-d_{2}\right)\right) \\
& +\alpha T e^{r_{f}-\rho \sigma_{S} \sigma_{C}}\left[\Phi\left(d_{2}-\sigma_{S}\right)-\Phi\left(d_{1}-\sigma_{S}\right)\right] \text {, }
\end{aligned}
$$

where $d_{1}, d_{2}$ are defined in (11) and (12).

Now, we are ready to test some numerical examples. We begin with a description of the parameters of Black-Scholes quanto model for the VA contracts: the contract maturity $T=5$, initial investment $P=100$, floor rate $f=1 \%$, the volatility of the linked-index $\sigma_{s}=25 \%$, the volatility of the exchange rate $\sigma_{C}=10 \%$, the correlation coefficient $\rho=-0.1$, local currency interest rates $r=6 \%$, foreign currency interest rates $r_{f}=4 \%$, and the global guarantee rate $G=110 \%$.

We simulate 1000 independent runs of $\left(\tilde{R}_{1}, \cdots, \tilde{R}_{T}\right)$. From these 1000 simulated paths, we can easily obtain 1000 independent replications of $e^{-r T} P \cdot \max \left(R_{M}, G\right)$. Based on these independent copies, standard point estimates of $E\left[e^{-r T} P \cdot \max \left(R_{M}, G\right)\right]$, and their standard errors are computed and presented in Table 2.

Table 2: Fair value of the VA contract computed by naive Monte carlo method. The upper table contains point estimates and the lower table contains their standard errors.

\begin{tabular}{|r|rrrrr|}
\hline$\alpha \backslash c$ & 0.10 & 0.15 & 0.20 & 0.30 & 0.40 \\
\hline 0.6 & 90.81 & 95.26 & 98.34 & 101.77 & 103.20 \\
0.8 & 91.78 & 97.37 & 101.60 & 107.26 & 110.28 \\
1.0 & 92.42 & 98.69 & 103.86 & 111.26 & 116.01 \\
1.2 & 92.86 & 99.60 & 105.43 & 114.34 & 120.50 \\
1.4 & 93.17 & 100.30 & 106.56 & 116.74 & 124.11 \\
1.6 & 93.41 & 100.84 & 107.45 & 118.63 & 127.09 \\
1.8 & 93.60 & 101.25 & 108.17 & 120.12 & 129.58 \\
2.0 & 93.76 & 101.59 & 108.77 & 121.30 & 131.64 \\
\hline \hline 0.6 & 0.199 & 0.288 & 0.361 & 0.462 & 0.517 \\
0.8 & 0.208 & 0.310 & 0.395 & 0.533 & 0.626 \\
1.0 & 0.212 & 0.322 & 0.418 & 0.578 & 0.701 \\
1.2 & 0.214 & 0.329 & 0.433 & 0.609 & 0.754 \\
1.4 & 0.216 & 0.334 & 0.443 & 0.634 & 0.792 \\
1.6 & 0.218 & 0.336 & 0.450 & 0.652 & 0.823 \\
1.8 & 0.219 & 0.338 & 0.455 & 0.666 & 0.848 \\
2.0 & 0.220 & 0.340 & 0.457 & 0.676 & 0.868 \\
\hline
\end{tabular}

The accuracy of the point estimates in Table 2 are not very satisfactory. We shall apply the variance reduction technique of control variate. In particular, we take advantage of Proposition 1 and select control variate

$$
C=R_{M}-E_{Q}\left[R_{M}\right],
$$

Using the same 1000 replications of $\left(\tilde{R}_{1}, \cdots, \tilde{R}_{T}\right)$, we can also obtain 1000 independent replications of $C$. Let $\lambda_{1}$ and $\lambda_{2}$ be any real numbers and set

$$
Y(\lambda)=e^{-r T} P \cdot \max \left(R_{M}, G\right)-\lambda C
$$

Since $E_{Q}[C]=0$, it is easy to see that $E[Y(\lambda)]$ equal to the fair value of the VA contract. Therefore, it provides alternative mean of computing the price. It is well known that the optimal (variance-minimizing) weight $\lambda^{*}$ of the control variate is $\operatorname{Cov}(Y, C) / \operatorname{Var}(C)$ (Bratley, Fox, and Schrage 1983). The quantities of $\operatorname{Cov}(Y, C)$ and $\operatorname{Var}(C)$ are estimated by the sample covariance and variance.

It turns out that control variate $Y(\lambda)$ is quite effective. Table 3 show the results. These results indicate that the accuracy of the estimates has been improved significantly with control variate $C$.

Table 3: Fair value of the VA contract computed by Monte carlo method with control variate $C=R_{M}-E_{Q}\left[R_{M}\right]$. The upper table contains point estimates and the lower table contains their standard errors.

\begin{tabular}{|r|rrrrr|}
\hline$\alpha \backslash c$ & 0.10 & 0.15 & 0.20 & 0.30 & 0.40 \\
\hline 0.6 & 90.74 & 95.17 & 98.22 & 101.59 & 102.99 \\
0.8 & 91.75 & 97.25 & 101.47 & 107.04 & 110.04 \\
1.0 & 92.39 & 98.60 & 103.69 & 111.08 & 115.72 \\
1.2 & 92.83 & 99.55 & 105.27 & 114.13 & 120.23 \\
1.4 & 93.15 & 100.25 & 106.46 & 116.49 & 123.86 \\
1.6 & 93.39 & 100.79 & 107.37 & 118.36 & 126.82 \\
1.8 & 93.58 & 101.21 & 108.10 & 119.87 & 129.25 \\
2.0 & 93.74 & 101.55 & 108.69 & 121.12 & 131.29 \\
\hline \hline 0.6 & 0.021 & 0.021 & 0.022 & 0.022 & 0.022 \\
0.8 & 0.020 & 0.020 & 0.021 & 0.021 & 0.021 \\
1.0 & 0.020 & 0.020 & 0.020 & 0.020 & 0.020 \\
1.2 & 0.019 & 0.019 & 0.020 & 0.020 & 0.020 \\
1.4 & 0.019 & 0.019 & 0.019 & 0.019 & 0.020 \\
1.6 & 0.019 & 0.019 & 0.019 & 0.019 & 0.019 \\
1.8 & 0.019 & 0.019 & 0.019 & 0.019 & 0.019 \\
2.0 & 0.019 & 0.019 & 0.019 & 0.019 & 0.019 \\
\hline
\end{tabular}

To further quantify the effectiveness of control variate $C$, we define variance reduction ratio as follows.

$$
\operatorname{VRR}=\frac{\operatorname{Var}(\text { naive estimator })}{\operatorname{Var}(\text { estimator with control variate })}
$$

Because most of the computational effort was used to generate the sample paths of $\left(\tilde{R}_{1}, \cdots, \tilde{R}_{T}\right)$, the additional work needed to compute $C$ is minor. Therefore, it seems reasonable to use VRR as a proxy of computational gain. Table 4 shows that the quantities of variance reduction ratio range from 88 to 2070. This indicates control variate $C$ is very effective in reducing the estimator's variance. 
Table 4: Variance reduction ratio of control variate $C=$ $R_{M}-E_{Q}\left[R_{M}\right]$.

\begin{tabular}{|r|rrrrr|}
\hline$\alpha \backslash c$ & 0.10 & 0.15 & 0.20 & 0.30 & 0.40 \\
\hline 0.6 & 87.9 & 179.4 & 275.4 & 435.6 & 537.2 \\
0.8 & 104.9 & 228.3 & 366.5 & 647.4 & 875.2 \\
1.0 & 116.1 & 263.3 & 439.2 & 818.7 & 1184.1 \\
1.2 & 123.2 & 286.2 & 490.0 & 951.3 & 1432.8 \\
1.4 & 128.4 & 301.5 & 526.1 & 1059.6 & 1631.4 \\
1.6 & 132.4 & 312.5 & 553.5 & 1144.8 & 1799.3 \\
1.8 & 135.7 & 321.1 & 573.8 & 1213.4 & 1946.9 \\
2.0 & 138.3 & 328.6 & 589.2 & 1268.6 & 2069.6 \\
\hline
\end{tabular}

\section{CONCLUDING REMARKS AND FUTURE WORKS}

In this paper, we introduced a variable annuity contract in Asia markets. The variable annuity contract has quanto feature and embedded cliquet options. Such contracts are attractive to the investor because of their protection against downside risk and significant upside potential. We proposed a control variate for improving the computation efficiency of valuing such contracts. The numerical results show that the control variate is quite effective. This also suggests that Monte Carlo methods can be a very efficient computational tool for pricing complex insurance products.

Our study also provides computational tools for analyzing the trade-off among various parameters for such contracts. For example, the following information can be obtained from our study:

1. The effect of participation rate $\alpha$ on cost;

2. The effect of ceiling rate $c$ on cost;

3. The effect of floor rate $f$ on cost; and

4. The effect of the global guarantee rate $G$ on cost.

Finally, we suggest a few directions for future research:

1. Study the impact of correlation between linkedindex and exchange rate on price .

2. Expand the model to include issuer's credit risk.

\section{ACKNOWLEDGMENTS}

This research was supported by the National Science Council of Taiwan under contract number NSC97-2410-H-004-041MY2.

\section{REFERENCES}

Baxter, M., and A. Rennie. 1996. Financial Calculus: An Introduction to Derivative Pricing. Cambridge University Press.
Black, F., and M. Scholes. 1973. The pricing of options and corporate liabilities. Journal of Political Economy 81:637-59.

Bratley, P., B. L. Fox, and L. Schrage. 1983. A guide to simulation. New York: Springer-Verlag.

Gerber, H., and E. Shiu. 2003. Pricing lookback options and dynamic guarantees. North American Actuarial Journal 7(1):48-67.

Hardy, M. 2004. Ratchet equity indexed annuities. In 14th Annual International AFIR Colloquium.

Hardy, M. R. 2003. Investment guarantees: Modelling and risk management for equity-linked life insurance. Wiley, New York.

Harrison, J. M., and D. M. Kreps. 1979. Martingales and arbitrage in multiperiod security markets. Journal of Economics Theory 20:381-408.

Harrison, J. M., and S. R. Pliska. 1981. Martingales and stochastic integrals in the theory of continuous trading. Stochastic Processes and their Applications 11:215-60.

Hsieh, M.-H., and Y.-F. Chiu. 2007. Monte carlo methods for valuation of ratchet equity indexed annuities. In Proceedings of the 2007 Winter Simulation Conference, ed. S. Henderson, B. Biller, M.-H. Hsieh, and J. Shortle, 998-1003. Piscataway, New Jersey, USA: Institute of Electrical and Electronics Engineers, Inc.

Hull, J. C. 2006. Options, futures, and other derivatives securities, 6th edition. Prentice Hall International Editions.

Kjaer, M. 2006. Fast pricing of cliquet options with global floor. Journal Of Derivatives 14 (2): 47-60.

Law, A. M., and W. D. Kelton. 2000. Simulation modeling \& analysis. 3rd ed. New York: McGraw-Hill, Inc.

Lee, H. 2003. Pricing equity-indexed annuities with pathdependent options. Insurance, Mathematics, and Economics 33(3):677-690.

Tiong, S. 2000. Valuing equity-indexed annuities. North American Actuarial Journal 4:149-170.

\section{AUTHOR BIOGRAPHY}

MING-HUA HSIEH is an Associate Professor of the Department of Management Information Systems at National Chengchi University, Taiwan. From 1997 to 1999, he was a software designer at Hewlett Packard company, California. $\mathrm{He}$ is a member of INFORMS. His research interests include simulation methodology and financial engineering. He has served as a Proceedings co-editor for WSC'07. His e-mail address is $\langle$ mhsieh@nccu.edu.tw $>$. 